\title{
Immunotherapy in patients with early stage resectable nonsmall cell lung cancer
}

\author{
Katrien Ghysen and Johan Vansteenkiste
}

\begin{abstract}
Purpose of review
'Early-stage' nonsmall cell lung cancer (NSCLC) refers to stage I and stage II disease, and selected cases of stage IIIA disease where complete tumor resection is feasible. Surgery is the standard treatment for early NSCLC, but the overall 5-year survival remains below 50\%. The addition of adjuvant cisplatin-based chemotherapy to surgery improved 5-year survival rates by $5-10 \%$, but no significant therapeutic innovation has been established thereafter. We review recent and ongoing studies looking how immunotherapy may improve the outcome of these patients.
\end{abstract}

\begin{abstract}
Recent findings
Antigen-specific immunotherapy (cancer vaccination) did not fulfill its promise in a large phase III randomized trial in completely resected early-stage NSCLC. Over the past few years, immunotherapy with immune checkpoint inhibition $(\mathrm{ICl})$ has led to remarkable progress in nononcogene addicted metastatic NSCLC. We review ongoing clinical investigations that want to translate these benefits to earlier stages of NSCLC. Both adjuvant and neoadjuvant large randomized controlled trials with ICI are ongoing.
\end{abstract}

\begin{abstract}
Summary
Although from a mechanistic perspective the neoadjuvant administration may be preferred, it is crucial that both adjuvant and neoadjuvant trials are recruited as planned, to have a balanced view how ICl therapy may ultimately improve cure rates in these patients.
\end{abstract}

Keywords

adjuvant, early stage, immune checkpoint inhibition, neoadjuvant, nonsmall cell lung cancer

\section{INTRODUCTION}

Nonsmall cell lung cancer (NSCLC) represents $85 \%$ of all lung cancers. The prognosis and the treatment approach are highly dependent on a proper staging of the extent of the disease. Surgical resection is the cornerstone treatment for patients with stage I and II disease and selected patients with stage IIIA disease with a possibility of complete tumor resection. The expected 5-year survival of this small group of early-stage NSCLC ranges from 90 to 41\%, according to the pathological stage [1]. Adjuvant chemotherapy is currently recommended for completely resected stages II and IIIA disease and can be considered in patients with resected stage IB disease and a primary tumor more than $4 \mathrm{~cm}$. If single station N2 disease can be demonstrated by preoperative pathological nodal staging, both definitive chemoradiotherapy and induction therapy followed by surgery are options, with surgery preferably considered in patients in whom a complete resection by lobectomy is expected [2].

\section{CURRENT ADJUVANT STRATEGIES}

The frequency of postoperative distant recurrences - associated with significantly reduced survival - is increasing according to tumor stages, ranging from $15 \%$ in stage IA to $60 \%$ in stage IIIA [3]. The aim of adjuvant therapy is to eliminate sparse tumor cells remaining after surgery and thereby reducing the recurrence rate. The International Adjuvant Lung cancer Trial in 2004 - where adjuvant cisplatinbased chemotherapy was associated with a statistically significant $4.1 \%$ 5-year survival advantage has brought adjuvant cisplatin-based doublet

Respiratory Oncology Unit, Department of Respiratory Medicine, University Hospitals KU Leuven, Leuven, Belgium

Correspondence to Johan Vansteenkiste, Respiratory Oncology Unit, Department of Respiratory Medicine, University Hospitals KU Leuven, Herestraat 49, B-3000 Leuven, Belgium. Tel: +32 16 346809;

e-mail: johan.vansteenkiste@uzleuven.be; web: www.llcg.be

Curr Opin Oncol 2018, 30:000-000

DOI:10.1097/CCO.0000000000000497 


\section{KEY POINTS}

- Surgery is the standard treatment for early-stage NSCLC, but the overall 5-year survival remains below $50 \%$ and no significant therapeutic innovation has been established since the introduction of adjuvant chemotherapy in 2004.

- A large randomized controlled trial with MAGE-A3directed antigen-specific immunotherapy could not confirm the hope that vaccines would be a step forward.

- The major progress with $\mathrm{ICl}$ targeting the programmed death 1/PD-L1 pathway in metastatic NSCLC, and the recent success of consolidation $\mathrm{ICl}$ in unresectable stage III NSCLC are encouraging signals to study $\mathrm{ICl}$ in early stage resectable NSCLC.

- There is preclinical evidence that neoadjuvant $\mathrm{ICl}$ could be more efficient than adjuvant immunotherapy.

- It is crucial that both adjuvant and neoadjuvant ICI trials are fully recruited as planned to have a balanced view how $\mathrm{ICl}$ therapy may ultimately improve cure rates in early-stage NSCLC.

chemotherapy as the standard of care for completely resected stage II and IIIA NSCLC. [4]. No further innovations in adjuvant therapy have been made since then. The role of cisplatin-pemetrexed versus cisplatin-vinorelbine was evaluated in the phase II randomized TREAT study [5]. Although the pemetrexed-based therapy was better tolerated, there were no signs of improvement of 3-year survival in a follow-up report [6]. A large phase III study on this question is fully recruited and results are awaited (JIPANG trial UMIN000006737) [7]. The addition of bevacizumab did not improve survival in the Eastern Cooperative Oncology Group 1505 study [8] nor did erlotinib in the RADIANT (A study of Erlotinib (Tarceva) after surgery with or without adjuvant chemotherapy in non-small cell lung carcinoma (NSCLC) patients who have Epidermal Growth Factor Receptor (EGFR) positive tumors) study [9].

\section{ADJUVANT IMMUNOTHERAPY}

\section{Antigen-specific immunotherapy (cancer vaccination)}

Because the standard adjuvant therapy - cisplatinbased chemotherapy - is often poorly tolerated after thoracic surgery, therapeutic vaccines with minimal toxicity have been studied for their improvement of outcome in the postresection setting with often minimal residual tumor. The melanoma-associated antigen (MAGE)-A3 is a tumor-specific antigen and is present on the surface of NSCLC cells (30-50\%). The MAGE-A3 vaccine (a recombinant proteinbased vaccine based on the full MAGE-A3 protein combined with a strong immunostimulant) has been studied in the large phase III double-blind, randomized, placebo-controlled MAGRIT (MAGEA3 as adjuvant non-small cell lung cancer immunotherapy) trial. A total of 2272 patients with resected stage IB-IIIA MAGE-A3-positive NSCLC, who could receive adjuvant chemotherapy according to standard practice, were randomly assigned (2:1) to the MAGE-A3 immunotherapeutic or placebo during 27 months [10]. The primary end point, disease-free survival (DFS) was unfortunately not improved. Based on the current insights in tumor immunology, one can hypothesize that the truly-generated MAGE-A3-directed lymphocytes were probably not able to achieve their tumor cell killing properties, as they were inactivated by checkpoint interactions in the tumor microenvironment.

\section{Adjuvant immune checkpoint inhibition}

Based on the revolutionary results of immune checkpoint inhibition (ICI) in metastatic NSCLC, exploration in early-stage NSCLC was a logical next step. The interest in ICI for nonmetastatic NSCLC was recently further enhanced by the remarkable success of durvalumab ICI in stage III unresectable NSCLC [11"].

Four large randomized controlled phase III trials are currently investigating the use of ICI as adjuvant treatment after surgical resection (Table 1): PEARLS

Table 1. Ongoing phase III trials of adjuvant immunotherapy in stage I-IIIA NSCLC

\begin{tabular}{lllcl}
\hline Study (identifier) & Sponsor & Compound & Target accrual & Primary end point \\
\hline PEARLS (NCT02504372) & EORTC/ETOP and MSD & Pembrolizumab & 1380 & DFS \\
ANVIL (NCT02595944) & NCl and BMS & Nivolumab & 714 & DFS and OS \\
BR31 (NCT02273375) & NCl-C and AZ & Durvalumab & 1100 & DFS in PD-L1 positive \\
IMpower-010 (NCT02486718) & Hoffman-La Roche & Atezolizumab & 1127 & DFS and DFS in PD-L1 positive \\
\hline
\end{tabular}

AZ, AstraZeneca; BMS, Bristol-Myers Squibb; DFS, disease-free survival; EORTC, European Organisation for Research and Treatment of Cancer; ETOP, European Thoracic Oncology Platform; MSD, Merck Sharp \& Dohme; NCl, National Cancer Institute; OS, overall survival; PD-Ll, programmed death ligand 1. 
(pembrolizumab versus placebo for patients with early-stage NSCLC after resection and completion of standard adjuvant therapy, NCT02504372), Canadian cancer trial groups BR31 (NCT02273375), ANVIL (adjuvant nivolumab in resected lung cancers, NCT02595944), and IMpower-010 (NCT02486718). Although each of these trials has its merits, they also illustrate how each pharma partner is replicating the same question. All trials are in patients with completely resected stage IB more than $4 \mathrm{~cm}$, II, or IIIA, and allow adjuvant chemotherapy as per standard practice. Most allow resected tumors of any programmed death ligand 1(PD-L1) status, but the BR31 trial will enrich the trial population with PD-L1 positive tumors after enrolment of 600 patients. Two trials are placebo-controlled, whereas the ANVIL and IMpower-010 are not. DFS is the primary end point in PEARLS, for the BR31 this is DFS in PD-L1 positive tumors, IMpower-010 has both of these endpoints, and ANVIL targets DFS and overall survival.

The success of ICI therapy in advanced NSCLC is based on the interaction between tumor cells, antigen-presenting cells, and cytotoxic T lymphocytes, from which the inhibition by the programmed death $1 /$ PD-L1 pathway is taken away anti-programmed death 1/PD-L1 monoclonal antibodies. Therefore, potential disadvantages of adjuvant ICI administration are the paucity of remaining tumor cells after compete resection, hence the lower tumor burden and presentation of tumor neoantigens, and the disturbed locoregional lymph node function after the lymph node dissection during surgical resection. Moreover, as there are no early surrogate end points in the adjuvant setting, it will take time before this question will be answered, as the readouts of these trials are expected from 2021 onwards.

\section{NEOADJUVANT IMMUNE CHECKPOINT INHIBITION IMIMUNOTHERAPY}

Although resection of the primary tumor is indispensable for tumor control in early-stage lung cancer, the surgical procedure itself has been suspected to promote the postoperative recurrence by inducing perioperative dissemination of micrometastases, clearing tumor-derived antiangiogenic signals, and evoking the secretion of tumor growth factors and inducing postoperative cell-mediated immunosuppression [12]. Hence, neoadjuvant therapy - used to reduce tumor vitality and early action on micrometastatic disease - is an attractive strategy, even if this may give a delay in the surgical procedure. Even in early-stage NSCLC, there is an immunosuppressive tumor microenvironment with increased regulatory $\mathrm{T}$ cells and reduced natural killer cells in the tumor environment when compared with normal lung tissue [13], an additional reason why early repriming of the immune system may be beneficial. In the neoadjuvant setting, when the tumor mass and locoregional lymph nodes are intact, T-cell invigoration with ICI therapy seems particularly attractive.

Adjuvant and neoadjuvant ICI therapy was studied in a very nice Australian study with murine models of triple-negative breast cancer [14"]. Both adjuvant and neoadjuvant checkpoint inhibition resulted in increased levels of tumor-specific CD8 ${ }^{+}$ $\mathrm{T}$ lymphocytes in the tumor and in the peripheral blood, compared to controls. In the neoadjuvant administration, however, tumor-specific $\mathrm{CD}^{+}$levels were significantly higher in both the tumor and circulation. Moreover, these cells exhibited memory cell characteristics, hence, a prolonged antitumor activity, a feature of great interest in the postsurgical setting with micrometastatic disease. As a result, this led to a much longer animal survival when neoadjuvant immunotherapy was given in comparison to adjuvant administration.

At the meeting of the European Society of Medical Oncology in 2016, a first pilot study in 21 patients with resectable NSCLC was presented, and published thereafter [15"']. Two preoperative doses of the anti-programmed death 1 inhibitor nivolumab $(3 \mathrm{mg} / \mathrm{kg})$ were administered intravenously q2 weeks, with surgery planned about 4 weeks after start if the neoadjuvant therapy. First, neoadjuvant ICI therapy did not delay the surgical intervention. A major pathological response was seen in 9/20 complete resections. At the systemic

Table 2. Ongoing phase III trials of neoadjuvant immunotherapy in stage I-IIIA NSCLC

\begin{tabular}{|c|c|c|c|c|}
\hline Study (identifier) & Sponsor & Compound & Target accrual & Primary end point \\
\hline CheckMate 816 (NCT02998528) & BMS & Nivolumab \pm ipilimumab & 642 & EFS and PCR \\
\hline $\begin{array}{l}\text { MK-3475-671/KEYNOTE-671 } \\
\text { (NCT03425643) }\end{array}$ & MSD & Pembrolizumab & 786 & EFS and OS \\
\hline IMpower-030 (NCT03456063) & Hoffmann-La Roche & Atezolizumab & 302 & MPR \\
\hline
\end{tabular}

BMS, Bristol-Myers Squibb; EFS, event-free survival; MPR, major pathological response; MSD, Merck Sharp \& Dohme; OS, overall survival; PCR, pathological complete response. 
level, in these patients, tumor neoantigen-specific T-cell clones were found in the peripheral blood after neoadjuvant treatment. This means that most of the interesting observations in the Australian preclinical study could be replicated in preoperative NSCLC patients.

Based on this, several dedicated academic clinical trials and large phase III trials with a neoadjuvant ICI therapy strategy have been initiated (Table 2). The safety and effectiveness of nivolumab and ipilimumab or nivolumab and chemotherapy versus chemotherapy alone in early stage (IB-IIIA) operable NSCLC is evaluated in a randomized, open label, phase III trial, with major pathologic response (MPR) rate and DFS as primary end points. The surrogate end point MPR is anticipated to be reached in September 2020 (NCT02998528). A concern with this trial is that in one arm of this trial - ipilimumab/ nivolumab - the proven benefit of perioperative chemotherapy is omitted. This is not the case in the KEYNOTE-671 trial (NCT03425643). It is a randomized, placebo-controlled, double-blind phase III trial of concurrent neoadjuvant platinum doublet chemotherapy and pembrolizumab (four cycles) followed by surgery and adjuvant pembrolizumab (13 cycles) versus concomitant neoadjuvant platinum doublet chemotherapy and placebo (four cycles) followed by surgery and adjuvant placebo (13 cycles) for participants with resectable stage IIB or IIIA NSCLC. The IMpower-030 (NCT03456063) is a smaller trial that has the surrogate end point MPR as primary end point. Here as well, the proven benefit of perioperative chemotherapy is not omitted; patients with resectable stage II, IIA, and select IIIB NSCLC are randomly assigned to neoadjuvant atezolizumab and chemotherapy versus neoadjuvant placebo and chemotherapy.

\section{CONCLUSION}

The major progress with programmed death 1/PDL1 blockade in metastatic NSCLC could not yet be translated to the resectable early NSCLC setting, but the results of large phase III trials are now being awaited.

The signal from the unresectable stage III setting in the PACIFIC trial is most encouraging. A major progress in both progression-free survival [16] and overall survival [11"] with durvalumab as consolidation therapy after concurrent chemoradiotherapy was documented, making ICI consolidation the new standard of care in these patients. Both adjuvant and neoadjuvant ICI therapy are now being intensively studied in different phases of clinical trials. Preclinical data based on, for example, a murine mouse model for triple-negative breast cancer showed better efficacy of neoadjuvant immunotherapy over adjuvant immunotherapy. Moreover, neoadjuvant trials have the possibility to include surrogate end points such as MPR in their design, and will therefore have an earlier readout than the adjuvant trials. As there are no clinical trials that compare adjuvant versus neoadjuvant immunotherapy, it is vital that both ongoing adjuvant and neoadjuvant trials achieve full recruitment, so that surrogate end points will not be the only guide for future clinical practice.

\section{Acknowledgements}

None.

\section{Financial support and sponsorship}

This is an academic manuscript without any external support.

$J . V$. has a research funding at University Hospitals $K U$ Leuven by MSD, advisory functions and lectures for Apotex, AstraZeneca, Boehringer Ingelheim, MSD, Novartis, and Roche.

\section{Conflicts of interest}

There are no conflicts of interest.

\section{REFERENCES AND RECOMMENDED}

\section{READING}

Papers of particular interest, published within the annual period of review, have been highlighted as:

- of special interest

- of outstanding interest

1. Goldstraw $P$, Chansky K, Crowley J, et al. The IASLC lung cancer staging project: proposals for revision of the TNM stage groupings in the forthcoming (eighth) edition of the TNM classification for lung cancer. J Thorac Oncol 2016; 11:39-51.

2. Vansteenkiste J, De Ruysscher D, Eberhardt W, et al. Early and locally advanced nonsmall cell lung cancer (NSCLC): ESMO clinical practice guidelines for diagnosis, treatment and follow-up. Ann Oncol 2013; 24(Suppl 6):vi89-vi98.

3. Aliperti LA, Predina JD, Vachani A, Singhal S. Local and systemic recurrence is the Achilles heel of cancer surgery. Ann Surge Oncol 2011; 18:603-607.

4. Arriagada R, Bergman B, Dunant A, et al., The International Adjuvant Lung Cancer Trial Collaborative Group. Cisplatin-based adjuvant chemotherapy in patients with completely resected nonsmall cell lung cancer. $\mathrm{N}$ Engl J Med $2004 ; 350: 351-360$.

5. Kreuter M, Vansteenkiste JF, Fischer JR, et al., TREAT investigators. Randomized phase 2 trial on refinement of early-stage NSCLC adjuvant chemotherapy with cisplatin and pemetrexed versus cisplatin and vinorelbine: the TREAT study. Ann Oncol 2013; 24:986-992.

6. Kreuter M, Vansteenkiste J, Fischer JR, et al. Three-year follow-up of a randomized phase II trial on refinement of early stage NSCLC adjuvant chemotherapy with cisplatin and pemetrexed versus cisplatin and vinorelbine (the TREAT study). J Thorac Oncol 2016; 11:85-93.

7. Yamamoto N, Kenmotsu H, Yamanaka T, et al. Randomized phase III study of cisplatin with pemetrexed and cisplatin with vinorelbine for completely resected nonsquamous nonsmall cell lung cancer: the JIPANG study protocol. Clin Lung Cancer 2018; 19:e1-e3.

8. Wakelee HA, Dahlberg SE, Keller SM, et al., ECOG-ACRIN. Adjuvant chemotherapy with or without bevacizumab in patients with resected nonsmall cell lung cancer (E1505): an open-label, multicentre, randomised, phase 3 trial. Lancet Oncol 2017; 18:1610-1623.

9. Kelly K, Altorki NK, Eberhardt WE, et al. Adjuvant erlotinib versus placebo in patients with stage IB-IIIA nonsmall cell lung cancer (RADIANT): a randomized, double-blind, phase III trial. J Clin Oncol 2015; 33:4007-4014. 
10. Vansteenkiste JF, Cho BC, Vanakesa T, et al. Efficacy of the MAGE-A3 cancer immunotherapeutic as adjuvant therapy in patients with resected MAGE-A3positive nonsmall-cell lung cancer (MAGRIT): a randomised, double-blind, placebo-controlled, phase 3 trial. Lancet Oncol 2016; 17:822-835.

11. Antonia SJ, Villegas $A$, Daniel $D$, et al., PACIFIC Investigators. Overall survival

- with durvalumab after chemoradiotherapy in stage III NSCLC. N Engl J Med 2018; doi: 10.1056/NEJMoa1809697. [Epub ahead of print]

First randomized controlled trial that demonstrated a clinically relevant survival benefit with $\mathrm{ICl}$ therapy in nonmetastatic NSCLC, with the hope of improving cure rate.

12. Shakhar G, Ben-Eliyahu S. Potential prophylactic measures against postoperative immunosuppression: could they reduce recurrence rates in oncological patients? Ann Surge Oncol 2003; 10:972-992.

13. Lavin $Y$, Kobayashi $S$, Leader $A$, et al. Innate immune landscape in early lung adenocarcinoma by paired single-cell analyses. Cell 2017; 169:750-765.
14. Liu J, Blake SJ, Yong MC, et al. Improved efficacy of neoadjuvant compared to - adjuvant immunotherapy to eradicate metastatic disease. Cancer Discov $2016 ; 6: 1382-1399$.

This preclinical study showed a better efficacy of neoadjuvant immunotherapy over adjuvant immunotherapy. Tumor-specific $\mathrm{CD}^{+}$levels were significantly higher in the neoadjuvant administration which demonstrates a prolonged antitumor activity. This could be of great interest in the postoperative setting.

15. Forde PM, Chaft JE, Smith KN, et al. Neoadjuvant PD-1 blockade in resectable n lung cancer. N Engl J Med 2018; 378:1976-1986.

This pilot study proved a potential benefit of short-term neoadjuvant nivolumab before surgery for early-stage NSCLC. Based on this study, several large phase III trials with neoadjuvant $\mathrm{ICl}$ therapy have been initiated.

16. Antonia SJ, Villegas A, Daniel D, et al., PACIFIC Investigators. Durvalumab after chemoradiotherapy in stage III nonsmall cell lung cancer. N Engl J Med 2017; 377:1919-1929. 